\title{
Relationship between pulmonary function and brachial-ankle pulse wave velocity in coal miners in northern China
}

\author{
Xinhua Yan ${ }^{1}$, Lixia Sun ${ }^{2}$, Mengying $\mathrm{Ma}^{2}$, Jiqiang Liu ${ }^{3}$, Shouling $\mathrm{Wu}^{4}$, Jie Cao ${ }^{1}$ \\ ${ }^{1}$ General Hospital of Tianjin Medical University, Tianjin 300070, China; ${ }^{2}$ Emergency Department, North China University of Science and \\ Technology Affiliated Hospital, Tangshan 063009, China; ${ }^{3}$ Zhejiang Lishui Huaqiao Hospital, Lishui 323000, China; ${ }^{4}$ Department of Cardiology, \\ Kailuan General Hospital, Tangshan 063009, China \\ Contributions: (I) Conception and design: S Wu; (II) Administrative support: J Cao; (III) Provision of study materials or patients: J Liu, L Sun; (IV) \\ Collection and assembly of data: M Ma; (V) Data analysis and interpretation: X Yan; (VI) Manuscript writing: All authors; (VII) Final approval of \\ manuscript: All authors. \\ Correspondence to: Jie Cao: General Hospital of Tianjin Medical University, Tianjin 300070, China. Email: dr_caojie@sina.com; Shouling Wu. \\ Department of Cardiology, Kailuan General Hospital, Tangshan 063009, China. Email: drwus1@163.com.
}

Background: To investigate the relationship between pulmonary function and brachial-ankle pulse wave velocity (baPWV).

Methods: A cross-sectional study was conducted. A total of 11,388 people with complete pulmonary function test and baPWV data and who participated in both the health examination of the Kailuan Occupational Disease Prevention and Treatment Center in 2014-2016 and the health checkup of the Kailuan Group in 2012 and 2014 were selected as subjects. The study population was divided into four groups by forced vital capacity (FVC) quartiles (group 1: FVC <3.50 L; group 2: $3.50 \mathrm{~L} \leq \mathrm{FVC}<3.96 \mathrm{~L}$; group 3: $3.96 \mathrm{~L} \leq \mathrm{FVC}<4.47 \mathrm{~L}$; group 4: FVC $\geq 4.47 \mathrm{~L}$ ) and divided into four groups by forced expiratory volume in one second (FEV1) quartile (group 1: FEV1 <3.15 L; group 2: $3.15 \mathrm{~L} \leq \mathrm{FVC}<3.61 \mathrm{~L}$; group 3: $3.61 \mathrm{~L} \leq \mathrm{FVC}<4.08 \mathrm{~L}$; group 4: $\mathrm{FVC} \geq 4.08 \mathrm{~L}$ ). Linear regression analysis and multivariate logistic regression were used to analyze the effects of pulmonary function on baPWV.

Results: When grouped by FVC, the baPWV of the first group was significantly higher than the other groups. Similarly, the incidence of arteriosclerosis in the first group was significantly higher than the other groups. When grouped by FEV1, the baPWV of the first group was significantly higher than the other groups. The incidence of arteriosclerosis was also significantly higher in the first group than the other groups. After correcting for other confounding factors using linear regression, it was found that the effects of FVC and FEV1 on the study subject's baPWV were -23.84 and $-24.65 \mathrm{~L}$, respectively. Multivariate logistic regression analysis showed that when grouped by FVC quartile, the risk of arteriosclerosis was increased by $34 \%$ in group 1 compared with group 4 (OR: 1.34, 95\% CI: 1.17-1.52); the risk of arteriosclerosis was increased by $16 \%$ in group 2 compared with group 4 (OR: 1.16, 95\% CI: 1.03-1.31). When grouped by the FEV1 quartile, the risk of arteriosclerosis was increased by $25 \%$ in group 1 compared with group 4 (OR: 1.25, 95\% CI: 1.10-1.42).

Conclusions: Decreased pulmonary function is negatively correlated with baPWV and is an independent risk factor for arteriosclerosis.

Keywords: Pulmonary function; brachial-ankle pulse wave velocity (baPWV); forced vital capacity (FVC); forced expiratory volume in one second (FEV1)

Submitted Jun 05, 2019. Accepted for publication Oct 17, 2019.

doi: $10.21037 /$ jtd.2019.10.71

View this article at: http://dx.doi.org/10.21037/jtd.2019.10.71

(C) Journal of Thoracic Disease. All rights reserved. 


\section{Introduction}

Cardiovascular disease is the most fatal disease in the world, with high morbidity and mortality $(1,2)$. Arteriosclerosis is a risk factor for cardiovascular disease, and it is also a pathophysiological basis of myocardial infarction, stroke, and all-cause death (3-5). The brachial ankle-pulse wave velocity (baPWV) is a sensitive indicator for detecting arteriosclerosis $(6,7)$. Pulmonary function tests are an important method to evaluate the degree of airway patency and lung volume. Because of the close relationship between the respiratory system and the circulatory system, the relationship between pulmonary function and cardiovascular disease has been widely discussed. Studies by Tockman and Hyung found that pulmonary function decline is associated with the development of coronary artery disease (CAD), which serves as an independent risk factor for cardiovascular events $(8,9)$. Amaral et al. found that total lung capacity (TLC) and forced vital capacity (FVC) were negatively correlated with PWV (10). In addition, a prospective study by Charlotte E Bolton et al showed a negative correlation between FVC and forced expiratory volume in one second (FEV1) and PWV, both in middle-aged and elderly subjects. Whether pulmonary function is related to pulse wave velocity is rarely reported. So, this study analyzed the relationship between pulmonary function and PWV of coal miners in northern China, based on the data of Kailuan Research (Registration No.: ChiCTR-TNC-11001489).

\section{Methods}

\section{Study subjects}

This study used a cross-sectional study method. Inclusion criteria: (I) gender is not limited; (II) races are not limited; (III) in-service and retired miners who participated in the 2014-2016 Kailuan occupational disease prevention and treatment health examination and have complete pulmonary function test data; (IV) have complete baPWV data; (V) have taken part in the 2012 and 2014 Kailuan Group health checkups; (VI) agreed to participate in the study. Exclusion criteria: (I) age $>60$ years; (II) serious physical disability that precludes the examination; (III) loss of smoking history; (IV) research data missing.

The study protocol was approved by the Ethics committee of Tangshan Medical Ethics Society and written informed consent was obtained from each participant before data collection.

\section{Epidemiological data collection}

A unified epidemiological questionnaire was developed. The questionnaire was first filled out by individuals. Then the information was verified in person by trained doctors. The contents include demographic data, personal behavior habits (smoking and physical exercise, etc.), history of hypertension, diabetes, myocardial infarction, cerebral infarction and family history. A history of smoking is defined as an average daily intake of at least one cigarette per day for at least 1 year. Physical exercise is defined as a moderate-intensity exercise at least 3 days per week, more than 30 minutes each time.

\section{Physical examination}

Subjects were undressed, uncapped, and lightly dressed, using a calibrated RGZ-120 body mass scale to measure height (accurate to $1 \mathrm{~mm}$ ) and body mass (accurate to $0.1 \mathrm{~kg})$. Body mass index $(\mathrm{BMI})=$ weight $/$ height ${ }^{2}\left(\mathrm{~kg} / \mathrm{m}^{2}\right)$. Blood pressure measurement: No smoking, tea or coffee was allowed within $30 \mathrm{~min}$ and subjects were seated for 15 min before the blood pressure was measured. The right radial artery blood pressure was measured using a calibrated mercury sphygmomanometer. The first phase of Korotkoff sound was taken as the systolic pressure, and the fifth phase of Korotkoff sound was taken as the diastolic pressure. The measurements were taken 3 times, with a 1-2 min measurement interval, and the average reading scores were selected as final data. In addition, the maximum value of systolic blood pressure of the left and right radial artery was collected.

\section{Blood biochemical index}

All subjects fasted for at least $8 \mathrm{~h}$ before taking venous blood samples. $5 \mathrm{~mL}$ of venous blood was collected in an EDTA vacuum tube at 7:00 am to 9:00 am. After centrifugation at $3,000 \mathrm{~g}$ for $10 \mathrm{~min}$ at room temperature, the upper serum was taken to detect total cholesterol (TC), triglyceride (TG), low density lipoprotein cholesterol (LDL-C), uric acid (UA), fasting blood glucose (FBG), and hypersensitive C-reactive protein (CRP) within $4 \mathrm{~h}$. The above analysis was performed on the Hitachi 7600 automatic biochemical analyzer. The operation was strictly carried out according to the reagent manual, and quality control was carried out by a professional inspector. 


\section{baPWV determination}

The baPWV was measured using a BP-203RPEIII, PWV/ ABI type arteriosclerosis tester manufactured by Omron Corporation of Japan. The patient's age, sex, height, and body mass data were recorded prior to the examination. Before the examination, the participant was requested to rest for $15 \mathrm{~min}$ at room temperature. During the examination, the patient was lying on the examination bed, four oscillometric blood pressure cuffs were attached to the limbs. Simultaneously, the limb lead electrocardiogram electrode was connected, and a heart sound map PCG sensor was placed on the position of the electrocardiogram chest lead V4. Then the 4 cuffs were simultaneously inflated and deflated, and the ECG and PCG were monitored. The highest value of baPWV on the left and right sides was taken into the statistics. Arteriosclerosis was defined as baPWV $>1,400 \mathrm{~cm} / \mathrm{s}$.

\section{Pulmonary function test}

It was forbidden to eat a large amount of food $2 \mathrm{~h}$ before the test, drink cola, coffee, tea, etc. on the day of the test, smoke $1 \mathrm{~h}$ before the test or vigorously exercise $30 \mathrm{~min}$ before the test. During the test subjects were seated with their feet placed level on the ground. The examination was performed on a PTF lung function machine manufactured by the German company Jaeger. The tests were quality controlled and operated, and the participants were professionally instructed to ensure the accuracy of the results.

Test items include: FVC is the expiratory volume which requires inspiring to TLC position and exhaling as quickly and forcefully as possible to the residual volume (RV). FEV1 is the volume of air that is expired during the first second of a forced exhale after inspiration to the TLC.

Selection of test results: In three acceptable tests, the difference between the best and the suboptimal values of FVC and FEV1 should be $\leq 0.150 \mathrm{~L}$; if FVC $\leq 1.000 \mathrm{~L}$, the difference should be $\leq 0.100 \mathrm{~L}$. Both FVC and FEV1 take the maximum of all tests that meet the acceptable criteria and correct for the effect of individual body differences on lung function $(11,12)$. Correction formula: FVC $=$ FVC measured value $\times(1.73 / \text { height })^{2} ; \mathrm{FEV} 1=\mathrm{FEV} 1$ measured value $\times(1.73 / \text { height })^{2}$.

\section{Statistical analyses}

The physical examination data were entered by each hospital terminal, uploaded to the computer room server of Kailuan Hospital through the network, and the oracle database was formed, and verified by the cardiovascular laboratory staff of Kailuan Hospital. The Epidata database was established and processed by SPSS13.0 statistical software. According to the FVC and FEV1 quartiles, the mean of the measurement data among the four groups was compared by variance analysis, and the homogeneity of the variance and the normality test were performed. The data which satisfied with the normal distribution were showed as mean \pm standard error. The count data were showed as a percentage of $\mathrm{N}(\%)$. The ratio was compared using the $\chi^{2}$ test. As CRP and TG data were skewed, the data were then logarithmically transformed and compared between groups. Linear regression analysis and multivariate logistic regression model were used to analyze the effect of lung function on baPWV. To exclude the possible effects of smoking history, a sensitivity analysis was performed after excluding the history of smoking. The difference was considered statistically significant at $\mathrm{P}<0.05$ (two-sided test).

\section{Results}

\section{General data analysis}

According to the inclusion criteria, 12,743 people both took part in the 2014-2016 Open Occupational Disease Prevention and Treatment Hospital health check-up and participated in the 2012 and 2014 Kailuan Group health examinations. While, based on the exclusion criteria, one was missing baPWV and pulmonary function test data, 16 people over 60 years of age, 184 people with missing smoking history, and 1,154 people with missing biochemical indicator data were excluded. The final number of participants was 11,388 , which included 11,172 males and 216 females. The study population was divided into four groups by FVC quartiles (group 1: FVC $<3.50 \mathrm{~L}$; group 2: $3.50 \mathrm{~L} \leq \mathrm{FVC}<3.96 \mathrm{~L}$; group 3: $3.96 \mathrm{~L} \leq \mathrm{FVC}$ $<4.47 \mathrm{~L}$; group 4: FVC $\geq 4.47 \mathrm{~L})$. There were statistically significant differences in physical examination age, BMI, systolic blood pressure, fasting blood glucose, TC, HDL-C, LDL-C, $\lg$ TG, $\lg$ CRP, uric acid, baPWV, incidence of arteriosclerosis, hypertension, diabetes, and physical exercise (Table 1). The study population was also divided into four groups by FEV1 quartile (group 1: FEV1 $<3.15 \mathrm{~L}$; group 2: $3.15 \mathrm{~L} \leq \mathrm{FEV} 1<3.61 \mathrm{~L}$; group 3: $3.61 \mathrm{~L} \leq \mathrm{FEV} 1$ $<4.08 \mathrm{~L}$; group 4: FEV1 $\geq 4.08 \mathrm{~L}$ ), the difference between 
Table 1 General situation of different FVC level groups

\begin{tabular}{|c|c|c|c|c|c|}
\hline Characteristics & $\begin{array}{l}\text { Group } 1(<3.50) \\
\qquad(\mathrm{N}=2,796)\end{array}$ & $\begin{array}{c}\text { Group } 2(3.50-3.96) \\
\quad(N=2,918)\end{array}$ & $\begin{array}{c}\text { Group } 3(3.96-4.47) \\
\quad(N=2,859)\end{array}$ & $\begin{array}{l}\text { Group } 4(>4.47) \\
\quad(\mathrm{N}=2,815)\end{array}$ & $P$ value \\
\hline Age & $47.14 \pm 6.95$ & $43.45 \pm 8.21^{a}$ & $41.15 \pm 8.32^{\mathrm{ab}}$ & $36.86 \pm 8.33^{\mathrm{abc}}$ & $<0.001$ \\
\hline Systolic pressure $(\mathrm{mmHg})$ & $130.99 \pm 16.13$ & $128.80 \pm 15.23^{a}$ & $128.56 \pm 14.44^{\mathrm{a}}$ & $127.24 \pm 13.27^{\mathrm{abc}}$ & $<0.001$ \\
\hline Diastolic pressure (mmHg) & $83.60 \pm 10.32$ & $81.95 \pm 10.07^{\mathrm{a}}$ & $81.69 \pm 9.97^{a}$ & $80.20 \pm 9.48^{\mathrm{abc}}$ & $<0.001$ \\
\hline TC (mmol/L) & $5.19 \pm 1.04$ & $5.10 \pm 2.07^{\mathrm{a}}$ & $5.00 \pm 1.05^{\mathrm{ab}}$ & $4.85 \pm 1.13^{\mathrm{abc}}$ & $<0.001$ \\
\hline $\mathrm{HDL}(\mathrm{mmol} / \mathrm{L})$ & $1.36 \pm 0.78$ & $1.37 \pm 0.48$ & $1.39 \pm 0.60$ & $1.43 \pm 1.20^{\mathrm{ab}}$ & 0.015 \\
\hline LDL (mmol/L) & $2.80 \pm 0.88$ & $2.81 \pm 0.87$ & $2.79 \pm 0.95$ & $2.72 \pm 1.02^{\mathrm{abc}}$ & 0.002 \\
\hline BaPWV (cm/s) & $1,538 \pm 275$ & $1,469 \pm 246^{a}$ & $1,440 \pm 238^{\mathrm{ab}}$ & $1,378 \pm 200^{\mathrm{abc}}$ & $<0.001$ \\
\hline baPWV $\geq 1,400 \mathrm{~cm} / \mathrm{s}, \mathrm{n}(\%)$ & $1,857(66.4)$ & $1,630(55.9)$ & $1,462(51.1)$ & $1,139(40.5)$ & $<0.001$ \\
\hline Hypertension, n (\%) & $1,003(35.9)$ & $830(28.4)$ & $780(27.3)$ & $609(21.6)$ & $<0.001$ \\
\hline Diabetes, n (\%) & $278(9.9)$ & $203(7.0)$ & $161(5.6)$ & $95(3.4)$ & $<0.001$ \\
\hline History of smoking, n (\%) & $1,829(65.4)$ & $1,918(65.7)$ & $1,872(65.5)$ & $1,862(66.1)$ & 0.938 \\
\hline Physical exercise, n (\%) & 426 (15.2) & $310(10.6)$ & $259(9.1)$ & $181(6.4)$ & $<0.001$ \\
\hline
\end{tabular}

Data are presented as mean \pm SEM or number (percentage). Compared with the first quartile, ${ }^{a} \mathrm{P}<0.05$; compared with the second quartile, ${ }^{\mathrm{b}} \mathrm{P}<0.05$; compared with the third quartile, ${ }^{\mathrm{C}} \mathrm{P}<0.05$. FVC, forced vital capacity; BMI, body mass index; TC, total cholesterol; HDL, highdensity lipoprotein cholesterol; LDL, low-density lipoprotein cholesterol; baPWV, brachial-ankle pulse wave velocity; IgTG, log-transformed glycerol Triester; IgCRP, log-transformed hypersensitive C-reactive protein.

the groups did not change significantly (Table 2).

\section{Linear regression analysis}

A linear regression analysis method was applied, FVC was used as the dependent variable, baPWV was used as the independent variable. After correcting for physical examination age, BMI, CRP, LDL-C, TG, hypertension, diabetes, smoking history and physical exercise, the effect of FVC on baPWV in the study population was $-23.84 \mathrm{~cm} / \mathrm{s}$, the difference was statistically significant $(\mathrm{P}<0.001$, Table 3$)$. Moreover, FEV1 was negatively linearly correlated with baPWV $(\mathrm{P}<0.001$, Table 4).

\section{Multivariate logistic regression analysis}

Arteriosclerosis (baPWV $>1,400 \mathrm{~cm} / \mathrm{s}$ ) was used as the dependent variable and FVC quartile was used as independent variable in group 1 . Group 4 was used as baseline to perform the logistic regression analysis. After correcting for physical examination age, BMI, CRP, LDL-C, TG, hypertension, diabetes, smoking, physical exercise, our study showed that the risk of arteriosclerosis was increased by $34 \%$ in group 1 compared with group 4 (OR: 1.34 , 95\% CI: $1.17-1.52$, Table 5). The risk of arteriosclerosis increased by $16 \%$ in group 2 compared with group 4 (OR: 1.16, 95\% CI: 1.03-1.31, Table 5). Arteriosclerosis (baPWV $>1,400 \mathrm{~cm} / \mathrm{s}$ ) was used as the dependent variable, the FEV1 quartile was grouped as the independent variable, and group 4 was used as the baseline. Based on the logistic regression analysis, after adjusting for various confounding factors, the results showed that compared with group 4, the risk of developing arteriosclerosis in group 1 increased by $25 \%$ (OR: 1.25, 95\% CI: 1.10-1.42, Table 6). Sensitivity analysis was performed after excluding the smoking population, the results indicated that the risk still increased (Table 7). 
Table 2 General situation of different FEV1 level groups

\begin{tabular}{|c|c|c|c|c|c|}
\hline Characteristics & $\begin{array}{l}\text { Group } 1(<3.15) \\
\qquad(\mathrm{N}=2,812)\end{array}$ & $\begin{array}{c}\text { Group } 2(3.15-3.61) \\
\quad(\mathrm{N}=2,875)\end{array}$ & $\begin{array}{c}\text { Group } 3(3.61-4.08) \\
\quad(N=2,838)\end{array}$ & $\begin{array}{l}\text { Group } 4(>4.08) \\
\quad(\mathrm{N}=2,863)\end{array}$ & $P$ value \\
\hline Age (years) & $47.16 \pm 6.96$ & $43.35 \pm 8.26^{a}$ & $41.15 \pm 8.41^{\mathrm{ab}}$ & $37.01 \pm 8.25^{\mathrm{abc}}$ & $<0.001$ \\
\hline Systolic pressure $(\mathrm{mmHg})$ & $131.02 \pm 16.19$ & $128.74 \pm 15.11^{\mathrm{a}}$ & $128.42 \pm 14.54^{\mathrm{a}}$ & $127.42 \pm 14.86^{\mathrm{abc}}$ & $<0.001$ \\
\hline Diastolic pressure $(\mathrm{mmHg})$ & $83.50 \pm 10.32$ & $81.96 \pm 10.05^{\mathrm{a}}$ & $81.69 \pm 10.04^{a}$ & $80.31 \pm 9.47^{\mathrm{abc}}$ & $<0.001$ \\
\hline $\mathrm{TC}(\mathrm{mmol} / \mathrm{L})$ & $5.19 \pm 1.18$ & $5.10 \pm 2.01^{\mathrm{a}}$ & $5.00 \pm 0.97^{\mathrm{ab}}$ & $4.85 \pm 1.19^{\mathrm{abc}}$ & $<0.001$ \\
\hline $\mathrm{HDL}(\mathrm{mmol} / \mathrm{L})$ & $1.37 \pm 0.79$ & $1.38 \pm 0.60$ & $1.38 \pm 0.44$ & $1.43 \pm 1.19^{\mathrm{abc}}$ & 0.033 \\
\hline LDL (mmol/L) & $2.81 \pm 0.95$ & $2.80 \pm 0.95$ & $2.78 \pm 0.79$ & $2.73 \pm 1.01^{\mathrm{ab}}$ & 0.006 \\
\hline baPWV (cm/s) & $1,537 \pm 273$ & $1,468 \pm 250^{a}$ & $1,439 \pm 236^{\mathrm{ab}}$ & $1,382 \pm 200^{\mathrm{abc}}$ & $<0.001$ \\
\hline $\mathrm{baPWV} \geq 1,400 \mathrm{~cm} / \mathrm{s}$ & $1,859(66.1)$ & $1,587(55.2)$ & $1,449(51.1)$ & $1,193(41.7)$ & $<0.001$ \\
\hline Hypertension & $1,014(36.1)$ & $818(28.5)$ & $749(26.4)$ & $641(22.4)$ & $<0.001$ \\
\hline Diabetes & $264(9.4)$ & $209(7.3)$ & $159(5.6)$ & $105(3.7)$ & $<0.001$ \\
\hline History of smoking & $2,242(79.7)$ & $2,213(77.0)$ & $2,142(75.5)$ & $1,951(68.1)$ & $<0.001$ \\
\hline Physical exercise & $264(9.4)$ & $169(5.9)$ & $124(4.4)$ & $73(2.5)$ & $<0.001$ \\
\hline
\end{tabular}

Data are presented as mean \pm SEM or number (percentage). Compared with the first quartile, ${ }^{\mathrm{a}} \mathrm{P}<0.05$; compared with the second quartile, ${ }^{\mathrm{b}} \mathrm{P}<0.05$; compared with the third quartile, ${ }^{\mathrm{C}} \mathrm{P}<0.05$. FEV1, forced expiratory volume after 1 second; BMI, body mass index; $\mathrm{TC}$, total cholesterol; HDL, high-density lipoprotein cholesterol; LDL, low-density lipoprotein cholesterol; baPWV, brachial-ankle pulse wave velocity; IgTG, Number-converted triglyceride; IgCRP, log-transformed hypersensitive C-reactive protein.

\section{Discussion}

The respiratory system is closely related to the circulatory system. Many studies have shown that decreased lung function is an independent risk factor for cardiovascular disease $(2,3,13)$. Some studies suggest that arteriosclerosis may be the link between pulmonary dysfunction and cardiovascular disease. One diagnostic procedure $(4,5)$, baPWV, is an early sensitive indicator of arteriosclerosis, and its increase is an independent risk factor for cardiovascular disease. There are few reports on the relationship between lung function and baPWV.

In this study, coal miners in northern China were used as research subjects, and pulmonary function was measured by FVC and FEV1. We found that the level of baPWV was significantly higher in the low FVC group and the low FEV1 group $(\mathrm{P}<0.05)$. The higher the FVC and FEV1, the lower the incidence of arteriosclerosis (FVC: $66.4 \%$ in group 1 and $55.8 \%$ in group 2, $51.1 \%$ in 3 groups, $40.4 \%$ in group 4 ; FEV1: $66.1 \%$ in group $1,55.2 \%$ in group 2, $51 \%$ in group 3, $41.7 \%$ in group 4 ). In the low pulmonary function group, the incidence of arteriosclerosis was significantly higher than that in normal lung function group $(\mathrm{P}<0.05)$. There was a significant negative correlation between pulmonary function and baPWV. This is consistent with the findings of Bolton et al. (14).

In this study, we found through linear regression model that after adjusting for confounding factors, the effects of FVC and FEV1 on baPWV in the study population were -23.84 and $-25.58 \mathrm{~cm} / \mathrm{s}$, respectively, which is consistent with the results of Andre et al. A cross-sectional study was performed by them, showing that after adjusting for age, gender, height and race, the effects of TLC and FVC on PWV were -1.53 and $-2.66 \mathrm{~m} / \mathrm{s}$. In addition, continuing to adjust for smoking and BMI, the results showed no change. Meanwhile, in the logistic regression model, after 
Table 3 Linear regression analysis of the effect of FVC on baPWV $(\mathrm{n}=11,388)$

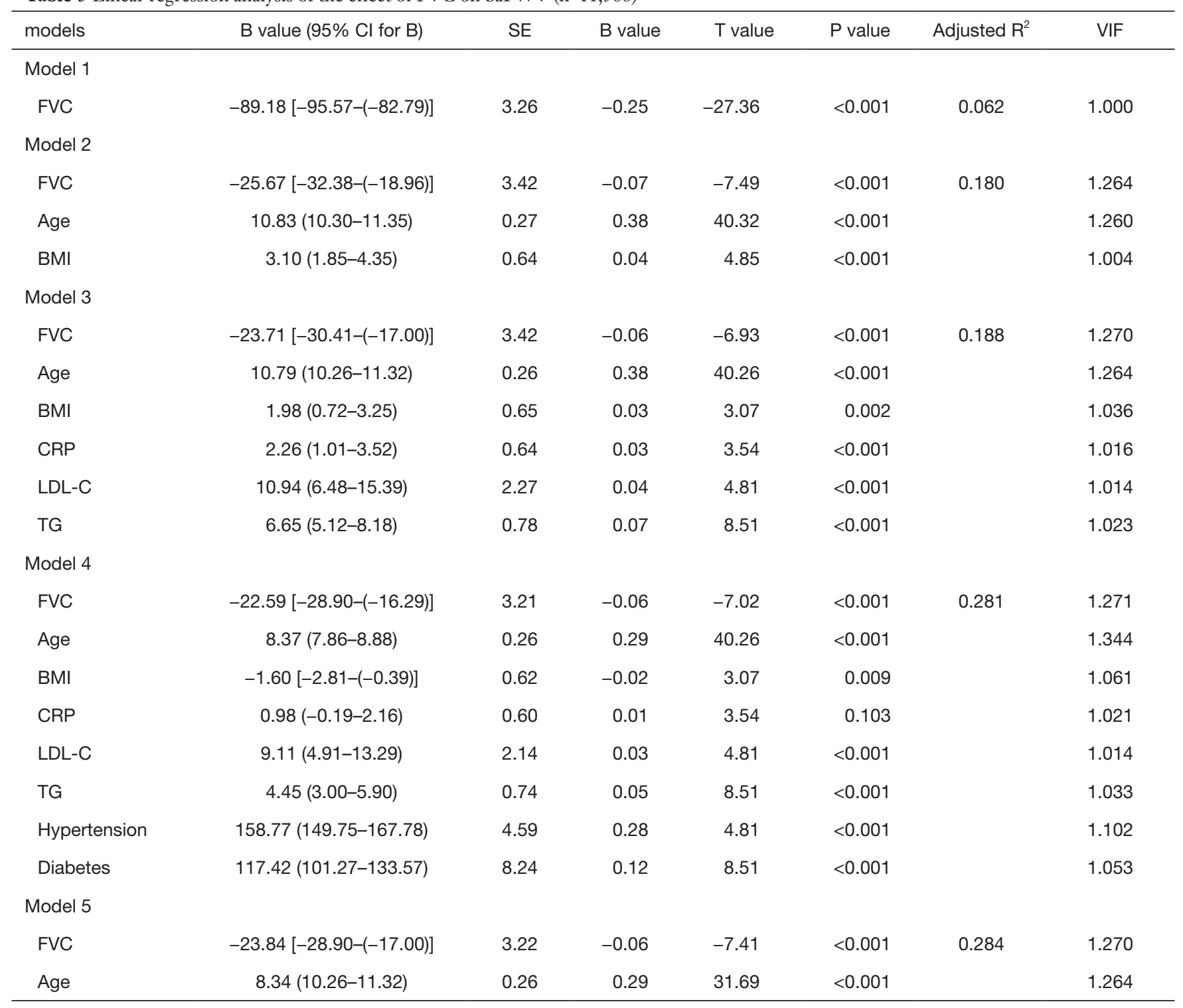

Model 1 uses baPWV as the dependent variable and FVC as the independent variable; Model 2 corrected for physical examination age and BMI; Model 3 further corrects for CRP, LDL-C, and TG based on Model 2; Model 4 corrects for hypertension or diabetes based on Model 3; Model 5 corrects for history of smoking and physical exercise based on Model 4. FVC, forced vital capacity; baPWV, brachial-ankle pulse wave velocity; BMI, body mass index; CRP, C-reactive protein; LDL, low-density lipoprotein cholesterol; TG, glycerol triester.

adjusting for confounding factors, the risk of arteriosclerosis in the lowest group of FVC (group 1) was 34\% higher than in the highest FVC group (group 4; OR: 1.34, 95\% CI: $1.25-1.63)$. The risk of arteriosclerosis was increased by $25 \%$ in the lowest FEV1 group (group 1) compared with the highest FEV1 group (group 4; OR: 1.25, 95\% CI: 1.17-1.52). This suggests that decreased pulmonary function can lead to an increased risk of arteriosclerosis and can be an independent risk factor for arteriosclerosis.
A prospective study conducted by Wannamethee et al. (12) showed that FVC and FEV1 were significantly negatively correlated with the incidence of fatal cardiovascular events, and decreased pulmonary function was an independent risk factor for cardiovascular disease, which is consistent with our study. Moreover, other studies showed that the decline in pulmonary function was associated with increased risk for cognitive dysfunction (15), microalbuminuria and acute kidney injury (16). A common pathological 
Table 4 Linear regression analysis of the effect of FEV1 on baPWV $(n=11,388)$

\begin{tabular}{|c|c|c|c|c|c|c|c|}
\hline Models & $\mathrm{B}$ value $(95 \% \mathrm{Cl}$ for $\mathrm{B})$ & SE & $B$ value & t value & $P$ value & Adjusted $\mathrm{R}^{2}$ & VIF \\
\hline FVC & $-90.76[-97.33-(-84.19)]$ & 3.34 & -0.25 & -27.09 & $<0.001$ & 0.061 & 1.000 \\
\hline \multicolumn{8}{|l|}{ Model 2} \\
\hline FVC & $-27.27[-34.12-(-20.43)]$ & 3.49 & -0.07 & -7.80 & $<0.001$ & 0.180 & 1.247 \\
\hline BMI & $3.14(1.89-4.39)$ & 0.64 & 0.04 & 4.92 & $<0.001$ & & 1.003 \\
\hline \multicolumn{8}{|l|}{ Model 3} \\
\hline FVC & $-25.56[-32.39-(-18.72)]$ & 3.48 & -0.07 & -7.33 & $<0.001$ & 0.188 & 1.251 \\
\hline Age & $10.78(10.25-11.29)$ & 0.26 & 0.38 & 40.46 & $<0.001$ & & 1.248 \\
\hline LDL-C & $11.00(6.55-15.45)$ & 2.27 & 0.04 & 4.84 & $<0.001$ & & 1.014 \\
\hline TG & $6.64(5.11-8.17)$ & 0.78 & 0.07 & 8.50 & $<0.001$ & & 1.023 \\
\hline \multicolumn{8}{|l|}{ Model 4} \\
\hline FVC & $-24.65[-31.08-(-18.22)]$ & 3.28 & -0.07 & -7.52 & $<0.001$ & 0.282 & 1.251 \\
\hline Age & $8.34(7.83-8.85)$ & 0.26 & 0.29 & 32.29 & $<0.001$ & & 1.328 \\
\hline BMI & $-1.58[-2.78-(-0.37)]$ & 0.62 & -0.02 & -2.57 & 0.010 & & 1.060 \\
\hline CRP & $1.03(-0.19-2.16)$ & 0.60 & 0.01 & 1.71 & 0.087 & & 1.020 \\
\hline LDL-C & $9.17(4.98-13.36)$ & 2.14 & 0.03 & 4.29 & $<0.001$ & & 1.014 \\
\hline Age & $8.32(7.81-8.84)$ & 0.26 & 0.29 & 31.86 & $<0.001$ & & 1.363 \\
\hline BMI & $-1.37[-2.58-(-0.17)]$ & 0.61 & -0.02 & -2.24 & 0.025 & & 1.063 \\
\hline CRP & $1.02(-0.16-2.19)$ & 0.60 & 0.01 & 1.69 & 0.090 & & 1.020 \\
\hline LDL-C & $8.75(4.57-12.94)$ & 2.13 & 0.03 & 4.10 & $<0.001$ & & 1.016 \\
\hline TG & $4.26(2.82-5.71)$ & 0.74 & 0.05 & 5.78 & $<0.001$ & & 1.035 \\
\hline Hypertension & $158.17(149.18-167.17)$ & 4.59 & 0.29 & 34.46 & $<0.001$ & & 1.103 \\
\hline Diabetes & $116.69(100.56-132.81)$ & 8.23 & 0.12 & 14.18 & $<0.001$ & & 1.054 \\
\hline History of smoking & $23.26[-32.54-(-6.75)]$ & 4.17 & 0.04 & 5.57 & $<0.001$ & & 1.014 \\
\hline Physical exercise & $-19.65[-32.01-(-19.15)]$ & 6.57 & -0.02 & -2.98 & 0.003 & & 1.034 \\
\hline
\end{tabular}

Model 1 uses baPWV as the dependent variable and FEV1 as the independent variable; Model 2 corrects for physical examination age and BMI; Model 3 further corrects for CRP, LDL-C, and TG based on Model 2; Model 4 corrects for hypertension or diabetes which is based on Model 3; Model 5 corrects for history of smoking and physical exercise based on Model 4. FEV1, forced expiratory volume after 1 second; baPWV, brachial-ankle pulse wave velocity; BMI, body mass index; LDL, low-density lipoprotein cholesterol; TG, triglyceride; CRP, C-reactive protein. 
Table 5 Logistic regression model of the influence of different FVC groups on baPWV $(>1,400 \mathrm{~cm} / \mathrm{s})$

\begin{tabular}{|c|c|c|c|c|c|}
\hline Variable & \multicolumn{5}{|c|}{ OR $(95 \% \mathrm{Cl})$} \\
\hline Group 4 (>4.08) & - & - & - & - & - \\
\hline Group $1(<3.15)$ & $2.91(2.61-3.25)$ & $1.33(1.18-1.51)$ & $1.28(1.13-1.45)$ & $1.31(1.15-1.48)$ & $1.34(1.17-1.52)$ \\
\hline Group 2 (3.15-3.61) & $1.86(1.67-2.07)$ & $1.13(1.01-1.27)$ & $1.11(0.99-1.25)$ & $1.15(1.02-1.29)$ & $1.16(1.03-1.31)$ \\
\hline BMI & - & $1.04(1.03-1.05)$ & $1.03(1.02-1.04)$ & $1.00(0.99-1.01)$ & $1.00(0.99-1.03)$ \\
\hline CRP & - & - & $1.02(1.00-1.03)$ & $1.01(0.99-1.03)$ & $1.01(0.99-1.02)$ \\
\hline LDL-C & - & - & $1.16(1.10-1.23)$ & $1.15(1.08-1.21)$ & $1.14(1.08-1.20)$ \\
\hline TG & - & - & $1.07(1.04-1.09)$ & $1.04(1.02-1.06)$ & $1.03(1.01-1.06)$ \\
\hline History of smoking & - & - & - & - & $1.18(1.08-1.29)$ \\
\hline Physical exercise & - & - & - & - & $0.81(0.70-0.93)$ \\
\hline
\end{tabular}

Model 1 is based on whether baPWV $\geq 1,400 \mathrm{~cm} / \mathrm{s}$ (yes =1, no $=0$ ), FVC quartiles are grouped as independent variables, with group 4 as the baseline; Model 2 corrects for age, and BMI; Model 3 further corrects for CRP, LDL-C, and TG based on Model 2; Model 4 corrects for hypertension and diabetes based on Model 3; Model 5 corrects for smoking history and physical exercise based on Model 4 . FVC, forced vital capacity; baPWV, brachial-ankle pulse wave velocity; BMI, body mass index; CRP, C-reactive protein; LDL, low-density lipoprotein cholesterol; TG, glycerol triester.

Table 6 Logistic regression model of the effects of different FEV1 groups on baPWV $(>1,400 \mathrm{~cm} / \mathrm{s})$

\begin{tabular}{|c|c|c|c|c|c|}
\hline Variable & \multicolumn{5}{|c|}{ OR (95\%Cl) } \\
\hline Group 4 (>4.47) & - & - & - & - & - \\
\hline Group $1(<3.50)$ & $2.73(2.45-3.04)$ & $1.24(1.10-1.40)$ & $1.20(1.06-1.36)$ & $1.24(1.09-1.40)$ & $1.25(1.10-1.42)$ \\
\hline Group 2 (3.50-3.96) & $1.72(1.55-1.91)$ & $1.05(0.94-1.17)$ & $1.03(0.92-1.15)$ & $1.07(0.95-1.21)$ & $1.08(0.96-1.22)$ \\
\hline Group 3 (3.96-4.47) & $1.46(1.31-1.62)$ & $1.05(0.94-1.18)$ & $1.04(0.94-1.16)$ & $1.08(0.96-1.21)$ & $1.18(0.97-1.22)$ \\
\hline BMI & - & $1.04(1.03-1.05)$ & $1.03(1.02-1.04)$ & $1.00(0.99-1.02)$ & $1.00(0.99-1.03)$ \\
\hline CRP & - & - & $1.02(1.01-1.04)$ & $1.02(0.99-1.03)$ & $1.01(0.99-1.03)$ \\
\hline LDL-C & - & - & $1.17(1.11-1.23)$ & $1.15(1.09-1.21)$ & $1.14(1.08-1.21)$ \\
\hline TG & - & - & 1.07 (1.04-1.09) & $1.04(1.02-1.06)$ & $1.04(1.01-1.06)$ \\
\hline History of smoking & - & - & - & - & $1.18(1.08-1.28)$ \\
\hline Physical exercise & - & - & - & - & $0.81(0.71-0.93)$ \\
\hline
\end{tabular}

Model 1 is based on whether baPWV $\geq 1,400 \mathrm{~cm} / \mathrm{s}$ (yes = 1, no = 0), FEV1 quartiles are grouped as independent variables, with group 4 as the baseline; Model 2 corrects for age, and BMI; Model 3 further corrects for CRP, LDL-C, and TG based on Model 2; Model 4 corrects for hypertension and diabetes based on Model 3; Model 5 corrects for smoking history and physical exercise based on Model 4. FEV1, forced expiratory volume after 1 second; baPWV, brachial-ankle pulse wave velocity; BMI, body mass index; LDL, low-density lipoprotein cholesterol; TG, triglyceride; CRP, C-reactive protein. 
Table 7 Logistic regression model of the effects of different FVC groups on baPWV ( $>1,400 \mathrm{~cm} / \mathrm{s})$ (after excluding smokers)

\begin{tabular}{|c|c|c|c|c|c|}
\hline Variable & \multicolumn{5}{|c|}{ OR $(95 \% \mathrm{Cl})$} \\
\hline Group 4 (>4.47) & - & - & - & - & - \\
\hline Group $1(<3.50)$ & $3.27(2.85-3.75)$ & 1.39 (1.19-1.63) & $1.32(1.13-1.55)$ & $1.34(1.14-1.57)$ & $1.34(1.14-1.58)$ \\
\hline Group 2 (3.50-3.96) & $1.98(1.74-2.25)$ & $1.14(0.98-1.31)$ & $1.12(0.97-1.29)$ & $1.16(0.99-1.34)$ & $1.16(1.00-1.35)$ \\
\hline Age & - & $1.08(1.07-1.09)$ & 1.08 (1.08-1.09) & 1.07 (1.06-1.08) & 1.07 (1.06-1.08) \\
\hline BMI & - & $1.03(1.02-1.05)$ & $1.02(1.00-1.04)$ & $0.99(0.97-1.01)$ & $0.99(0.98-1.01)$ \\
\hline CRP & - & - & 1.03 (1.01-1.05) & $1.02(1.00-1.04)$ & $1.02(1.01-1.04)$ \\
\hline LDL-C & - & - & $1.19(1.12-1.29)$ & $1.18(1.10-1.26)$ & $1.18(1.10-1.26)$ \\
\hline Diabetes & - & - & - & $2.21(1.72-2.86)$ & $2.21(1.71-2.86)$ \\
\hline Physical exercise & - & - & - & - & $0.88(0.75-1.05)$ \\
\hline
\end{tabular}

Model 1 is based on whether baPWV $\geq 1,400 \mathrm{~cm} / \mathrm{s}$ (yes =1, no = 0), FEV1 quartiles are grouped as independent variables, with group 4 as the baseline; Model 2 corrects for age, and BMI; Model 3 further corrects for CRP, LDL-C, and TG based on Model 2; Model 4 corrects for hypertension and diabetes based on Model 3; Model 5 corrects for physical exercise based on Model 4 . FVC, forced vital capacity; baPWV, brachial-ankle pulse wave velocity; BMI, body mass index; CRP, C-reactive protein; LDL, low-density lipoprotein cholesterol; TG, glycerol triester; FEV1, forced expiratory volume after 1 second.

feature of cardiovascular diseases, cognitive dysfunction, microalbuminuria, acute kidney injury and other diseases is arteriosclerosis. Thus, the decline in pulmonary function can be considered as an independent risk factor for developing arteriosclerosis and its accompanying comorbidities.

At present, the mechanism of arteriosclerosis caused by decreased pulmonary function may be as follows: (I) changes in pulmonary function and arterial function may be partly due to the same pathophysiological process which means under the influence of common risk factors, the two experience the same pathological process. Related studies have shown a significant correlation between decreased pulmonary function and hypertension (17), insulin resistance (18), impaired glucose tolerance and diabetes (19), and metabolic syndrome (MS). In this study, age, systolic blood pressure, fasting blood glucose, TC, TG, and CRP levels were significantly higher in the low FVC group than in the high FVC group, and the same pattern was observed in the low FEV1 group. It can be seen that there is a complex interaction between pulmonary function and various conventional cardiovascular risk factors, and common risk factors may lead to the decline of pulmonary function and arteriosclerosis simultaneously. (II) Hypoxia: patients with pulmonary diseases, especially those with chronic obstructive pulmonary disease, have chronic hypoxia, and chronic hypoxia has been shown to cause atherosclerosis through multiple effector pathways. In animal models, lack of oxygen can also induce atherosclerosis through the NF- $\kappa \mathrm{B}$ pathway and increase the oxidative stress marker lipid peroxide (20). (III) Chronic inflammatory response: a number of studies have shown that the level of inflammatory index C-reactive protein (CRP) is significantly increased in people with decreased pulmonary function and inflammation plays an important role in the occurrence and development of atherosclerotic lesions $(11,21,22)$. CRP can locally deposit in arteriosclerotic lesions during chronic inflammation, induce endothelial cells to express adhesion factors and chemotactic factors, activate monocytes to release inflammatory cytokines, and lead to atherothrombosis (23).

Limitations of this study: (I) this database is limited to employees in North China and Kailuan Group. Whether there will be the same results for different regions and different occupational groups, a larger sample of prospective studies is needed. (II) Only the 2014 annual brachial-ankle 
pulse wave velocity data were collected which means only the prevalence of arteriosclerosis can be calculated and not its incidence. (III) The study population is mainly male, whether gender will affect the results still needs to be further explored. However, due to the large sample size, this study still can explain the correlation between pulmonary function and pulse wave velocity in a certain degree.

In conclusion, our study revealed decreased pulmonary function was negatively correlated with baPWV and acts as an independent risk factor for arteriosclerosis. However, whether the relationship between decreased pulmonary function and arteriosclerosis leads to an increased risk of future cardiovascular disease requires further study.

\section{Acknowledgments}

None.

\section{Footnote}

Conflicts of Interest: The authors have no conflicts of interest to declare.

Ethical Statement: The authors are accountable for all aspects of the work in ensuring that questions related to the accuracy or integrity of any part of the work are appropriately investigated and resolved. The study protocol was approved by the Ethics committee of Tangshan Medical Ethics Society and written informed consent was obtained from each participant before data collection.

\section{References}

1. Vlachopoulos C, Aznaouridis K, Stefanadis C. Prediction of cardiovascular events and all-cause mortality with arterial stiffness: a systematic review and meta-analysis. J Am Coll Cardiol 2010;55:1318-27.

2. Amaral AF, Patel J, Gnatiuc L, et al. Association of pulse wave velocity with total lung capacity: A crosssectional analysis of the BOLD London study. Respir Med 2015;109:1569-75.

3. Tockman MS, Pearson JD, Fleg JL, et al. Rapid decline in FEV1. A new risk factor for coronary heart disease mortality. Am J Respir Crit Care Med 1995;151:390-8.

4. Sin DD, Wu L, Man SF. The relationship between reduced lung function and cardiovascular mortality: a population-based study and a systematic review of the literature. Chest 2005;127:1952-9.
5. Pac A, Tobiasz-Adamczyk B, Florek M, et al. Impaired lung function and mortality risk among older men and women. Findings from the 25-year follow-up study in Krakow, Poland. Eur Geriatr Med 2013;4:122.

6. Sabit R, Bolton CE, Edwards PH, et al. Arterial stiffness and osteoporosis in chronic obstructive pulmonary disease. Am J Respir Crit Care Med 2007;175:1259-65.

7. Lahousse L, van den Bouwhuijsen QJ, Loth DW, et al. Chronic obstructive pulmonary disease and lipid core carotid artery plaques in the elderly: the Rotterdam Study. Am J Respir Crit Care Med 2013;187:58-64.

8. Farro I, Bia D, Zocalo Y, et al. Pulse wave velocity as marker of preclinical arterial disease: reference levels in a uruguayan population considering wave detection algorithms, path lengths, aging, and blood pressure. Int J Hypertens 2012;2012:169359.

9. Bérard E, Bongard V, Ruidavets JB, et al. Pulse wave velocity, pulse pressure and number of carotid or femoral plaques improve prediction of cardiovascular death in a population at low risk. J Hum Hypertens 2013;27:529-34.

10. Amaral AF, Patel J, Gnatiuc L, et al. Association of pulse wave velocity with total lung capacity: A crosssectional analysis of the BOLD London study. Respir Med 2015;109:1569-75.

11. Cole TJ. Linear and Proportional Regression Models in the Prediction of Ventilatory Function. Journal of the Royal Statistical Society. Series A (General) 1975;138:297-338.

12. Wannamethee SG, Shaper AG, Rumley A, et al. Lung function and risk of type 2 diabetes and fatal and nonfatal major coronary heart disease events: possible associations with inflammation. Diabetes Care 2010;33:1990-6.

13. Sin DD, Man SF. Why are patients with chronic obstructive pulmonary disease at increased risk of cardiovascular diseases? The potential role of systemic inflammation in chronic obstructive pulmonary disease. Circulation 2003;107:1514-9.

14. Bolton CE, Cockcroft JR, Sabit R, et al. Lung function in mid-life compared with later life is a stronger predictor of arterial stiffness in men: the Caerphilly Prospective Study. Int J Epidemiol 2009;38:867-76.

15. Pathan SS, Gottesman RF, Mosley TH, et al. Association of lung function with cognitive decline and dementia: the Atherosclerosis Risk in Communities (ARIC) Study. Eur J Neurol 2011;18:888-98.

16. Yoon JH, Won JU, Ahn YS, et al. Poor lung function has inverse relationship with microalbuminuria, an early surrogate marker of kidney damage and atherosclerosis: 
the 5th Korea National Health and Nutrition Examination Survey. PLoS One 2014;9:e94125.

17. Jacobs DR Jr, Yatsuya H, Hearst MO, et al. Rate of decline of forced vital capacity predicts future arterial hypertension: the Coronary Artery Risk Development in Young Adults Study. Hypertension 2012;59:219-25.

18. Lazarus R, Sparrow D, Weiss ST. Baseline ventilatory function predicts the development of higher levels of fasting insulin and fasting insulin resistance index: the Normative Aging Study. Eur Respir J 1998;12:641-5.

19. Hickson DA, Burchfiel CM, Liu J, et al. Diabetes, impaired glucose tolerance, and metabolic biomarkers in individuals with normal glucose tolerance are inversely associated with lung function: the Jackson Heart Study. Lung 2011;189:311-21.

20. Song D, Fang G, Mao SZ, et al. Chronic intermittent

Cite this article as: Yan X, Sun L, Ma M, Liu J, Wu S, Cao J. Relationship between pulmonary function and brachial-ankle pulse wave velocity in coal miners in northern China. J Thorac Dis 2019;11(11):4551-4561. doi: 10.21037/jtd.2019.10.71 hypoxia induces atherosclerosis by NF-kappaB-dependent mechanisms. Biochim Biophys Acta 2012;1822:1650-9.

21. Gimeno D, Delclos GL, Ferrie JE, et al. Association of CRP and IL-6 with lung function in a middle-aged population initially free from self-reported respiratory problems: the Whitehall II study. Eur J Epidemiol 2011;26:135-44.

22. Hancox RJ, Gray AR, Sears MR, et al. Systemic inflammation and lung function: A longitudinal analysis. Respir Med 2016;111:54-9.

23. Patrick L, Uzick M. Cardiovascular disease: C-reactive protein and the inflammatory disease paradigm: HMGCoA reductase inhibitors, alpha-tocopherol, red yeast rice, and olive oil polyphenols. A review of the literature. Altern Med Rev 2001;6:248-71. 